\title{
Evaluation of oxidative stress in sheep infected with Psoroptes ovis using total antioxidant capacity, total oxidant status, and malondialdehyde level
}

\author{
Mustafa Sinan Aktas ${ }^{1}$, Fatih Mehmet Kandemir ${ }^{2}$, \\ Akin Kirbas ${ }^{1}$, Basak Hanedan ${ }^{1}$, Mehmet Akif Aydin ${ }^{3}$ \\ ${ }^{1}$ Department of Internal Medicine, ${ }^{2}$ Department of Biochemistry,Faculty of Veterinary Medicine, \\ ${ }^{3}$ Food and Livestock Applied Research Center, \\ Atatürk University, 25240, Erzurum, Turkey \\ akindahiliye55@yahoo.com
}

Received: January 3, $2017 \quad$ Accepted: May 12, 2017

\begin{abstract}
Introduction: The study aimed at evaluating oxidative stress using malondialdehyde (MDA), total antioxidant capacity (TAC), total oxidant status (TOS), and oxidative stress index (OSI) markers in sheep naturally infected with Psoroptes ovis (Acari). Material and Methods: The study was performed on 40 sheep divided into two equal groups: a healthy group (group I) and a group naturally infected with Psoroptes ovis (group II). The sera were obtained by centrifuging blood samples collected from the vena jugularis and serum MDA level changes in the samples were measured spectrophotometrically. Commercially available test kits were used for the measurement of TAC and TOS levels. The percentage ratio of TOS level to TAC level was accepted as OSI. Results: The serum malondialdehyde, total oxidant status levels, and oxidative stress index increased significantly $(\mathrm{P}<0.01)$ in group II, while the serum total antioxidant capacity levels decreased significantly $(\mathrm{P}<0.01)$ in this group. Negative correlations between total antioxidant capacity and total oxidant status and total antioxidant capacity and malondialdehyde, and a positive correlation between total oxidant status and malondialdehyde were found in infected sheep. Conclusion: The obtained results indicated the relationship between oxidant/antioxidant imbalance and Psoroptes ovis infection in sheep. Their MDA, TAC, TOS, and OSI markers may be used to determine the oxidative stress in natural infections with Psoroptes ovis.
\end{abstract}

Keywords: sheep, mange, Psoroptes ovis, oxidative stress.

\section{Introduction}

The ectoparasitic mite Psoroptes ovis causes sheep scab characterised by the rapid development of cutaneous inflammation and crusted skin lesions (35). The disease causes serious economic losses because of weight loss, skin and wool damage, lower milk production, worse pregnancy rate, weak lambs, increased susceptibility to other disease, and death (32).

A variety of inflammatory cells are activated to kill intra-cellular and extra-cellular parasites, inducing various oxidant-generating enzymes (24). Humoral and cellular immune response (IgG, $\operatorname{IgM}$, and especially $\operatorname{IgE}$ ) have a pivotal role in the pathogenesis of mange. Total leukocyte counts including T-lymphocyte, neutrophils, eosinophils, and mast cells, and globulin levels increase in animals with mange (19). Dermal mast cells have increased by $96 \mathrm{~h}$ after mange infection and Psoroptes ovis-specific IgE can be detected one week after infection (36). Alterations in the free radicals due to release of inflammatory cells may have a role in the pathogenesis of mange (19).

Free radicals, such as reactive oxygen and nitrogen species (ROS and RNS), are reactive chemical products which may cause oxidative damage by affecting macromolecules such as lipids, carbohydrates, proteins, and nucleic acids (15). Antioxidants play a role in preventing cell damage by reducing free radicals (23). There is a balance between free oxygen radicals and the preventive antioxidant system in 
healthy animals. The shift in the balance between free radicals and antioxidant system in favour of oxidants is known as oxidative stress. Oxidative stress is a part of cellular and molecular tissue damage in various diseases (15). Malondialdehyde (MDA), the last product of lipid peroxidation, enzymatic antioxidants such as superoxide dismutase, glutathione peroxidase, and catalase, non-enzymatic antioxidants such as reduced glutathione, vitamins $\mathrm{C}$ and $\mathrm{E}, \beta$-carotene, ceruloplasmin, and bilirubin, total antioxidant capacity (TAC), and total oxidant status (TOS) are widely used markers in the determination of oxidative stress in ruminants (9). Additionally, it is reported that the oxidative stress index (OSI) is a key factor for the determination of oxidative stress (5).

The determination of oxidative stress has become important recently in clinical practice as a complementary component (8). The occurrence of oxidative stress is proven under various conditions such as sepsis, mastitis, enteritis, respiratory and joint disease, endoparasitic diseases, transportation, and pregnancy in ruminants, and oxidative stress should be considered for the prognosis and effective treatment procedure of these conditions $(2,4,18,21,26,30)$. There are limited studies which evaluate oxidative stress in sheep mange $(13,19)$. Moreover, no study was found evaluating MDA, TAC, TOS, and OSI markers together in sheep with psoroptic mange. For this reason this study was aimed at evaluating oxidative stress using MDA, TAC, TOS, and OSI markers in sheep naturally infected with Psoroptes ovis (Acari).

\section{Material and Methods}

Animals and protocol design. This study was carried out in April and May 2014, in Erzurum city in eastern Anatolia, Turkey. The study material consisted of 40 female sheep, between 1 and 3 years of age, of Karayaka breed (Black Neck), housed in a semi-open barn, periodically treated with endoparasitic agents, and not exposed to excess insect stress (flies). The sheep were divided into two equal groups: a healthy group of infestation-free animals selected directly from the herd (group I) and the psoroptic mange group naturally infected with Psoroptes ovis, showing infection signs for $10 \pm 2$ days (group II). The clinical signs were recorded in both groups. Diagnosis of the disease was based on the clinical and parasitological examination findings.

Parasitological examination. Samples were obtained by skin scraping (scrapings varied from 1 to $2 \mathrm{~cm}^{2}$ ) until the skin was bleeding slightly, from a minimum of three different areas, and the samples were collected in dishes. The scraped samples were treated with $5 \mathrm{~mL}$ of $10 \%$ potassium hydroxide and two drops of this compound were put on a glass slide and examined under a microscope at $10 \times$ magnification (33).

Blood sampling. Blood samples of $10 \mathrm{~mL}$ were taken into sterile tubes (BD Vacutainer System, BD (Becton, Dickinson \& Company, UK) from the vena jugularis. Blood samples were kept at room temperature for $30 \mathrm{~min}$ and then centrifuged at $3,000 \mathrm{rpm}$ for $15 \mathrm{~min}$ and the serum was stored at $-20^{\circ} \mathrm{C}$ until analysis.

Biochemical assay. The changes in MDA levels in serum samples were measured spectrophotometrically by the method of Placer et al. (31) with modification. Commercially available test kits (Rel Assay Diagnostics, Turkey) were used for the measurement of TAC levels. The TAC levels were measured by a novel method based on automated colorimetric measurement developed by Erel (16). In the method, the hydroxyl radicals produced by the Fenton reaction react with o-dianisidine, which is a colourless substrate, to produce dianisyl radicals having bright yellowishbrown colour. After the serum sample was added, the oxidative reactions were initiated by the hydroxyl radicals in the reaction mixture and were suppressed by the antioxidant components of the serum. The result of this reaction was prevention of the colour change and thus the serum TAC levels were measured colourimetrically. The precision values of this assay are lower than 3\%. The analysis results are expressed as mmol Trolox equivalents/L.

Commercially available test kits (Rel Assay Diagnostics, Turkey) were used for the measurement of TOS levels. The TOS levels were measured by a novel method based on automated colorimetric measurement developed by Erel (17). Oxidants in the sample convert the ferrous ion-o-dianisidine complex to the ferric ion. Glycerol molecules abundantly present in the reaction mixture increase the oxidation reaction, and the ferric ion produces a coloured complex with xylenol orange in an acidic medium. The total amount of oxidant molecules in the sample determines the colour density and is measured spectrophotometrically. Hydrogen peroxide is used for the assay calibration and the analysis results are presented as $\mu$ mol $\mathrm{H}_{2} \mathrm{O}_{2}$ equivalent/L.

The percentage ratio of TOS level to TAC level was accepted as the OSI. The value of OSI was calculated according to the following formula: OSI $($ arbitrary unit $)=$ TOS $\left(\mu \mathrm{mol} \mathrm{H}_{2} \mathrm{O}_{2}\right.$ equivalent/L)/TAC (mmol Trolox equivalent/L) $(25,27)$.

Statistical analysis. The obtained data were tested using Student's $t$-test and the statistical package SPSS for Windows (1999), version 10.0 (IBM, USA). Means were considered significant at $\mathrm{P}<0.05$. Levene's test was used to test whether variances were homogenous. The relationship between MDA, TAC, TOS, and OSI in sheep infected with Psoroptes ovis was assessed by Pearson's correlation coefficient. 
Table 1. Serum levels of MDA, TAC, TOS, and OSI in healthy and psoroptic mange groups

\begin{tabular}{lll}
\hline Parameters & Healthy group (group I, $\mathrm{n}=20)$ & Psoroptic mange group (group II, $\mathrm{n}=20$ ) \\
\hline MDA (nmol/mL) & $2.98 \pm 0.56$ & $3.83 \pm 0.47^{* *}$ \\
TAC $(\mathrm{mmol}$ Trolox equivalent $/ \mathrm{L})$ & $1.54 \pm 0.22$ & $1.28 \pm 0.25^{* *}$ \\
TOS $\left(\mu \mathrm{mol} \mathrm{H}_{2} \mathrm{O}_{2}\right.$ equivalent $\left./ \mathrm{L}\right)$ & $1.89 \pm 0.27$ & $2.20 \pm 0.19^{* *}$ \\
OSI $($ arbitrary unit) & $1.25 \pm 0.24$ & $1.80 \pm 0.46^{* *}$ \\
\hline
\end{tabular}

MDA - malondialdehyde, TA - total antioxidant capacity, TOS - total oxidant status, OSI - oxidative stress index. The data are given as mean $\pm \mathrm{SE}$. $* * \mathrm{P}<0.001$ compared with healthy group

Table 2. Correlation between TAC, TOS, OSI, and MDA in psoroptic mange group

\begin{tabular}{lllll}
\hline & TAC & TOS & OSI & MDA \\
\hline TAC & - & -0.359 & $-0.932^{* *}$ & -0.006 \\
TOS & -0.359 & - & $0.622^{* *}$ & 0.305 \\
OSI & $-0.932^{* *}$ & $0.622^{* *}$ & - & 0.06 \\
MDA & -0.006 & 0.305 & 0.06 & - \\
\hline
\end{tabular}

MDA - malondialdehyde, TA - total antioxidant capacity, TOS - total oxidant status, OSI - oxidative stress index. $* * \mathrm{P}<0.01$

\section{Results}

Clinical signs. Clinical observations revealed alopecia, crusting, intense pruritus, and dermatitis occurring in different body areas in sheep of the psoroptic mange group. The healthy group had good body condition, appetite, and normal vital signs in the clinical examination.

Parasitological findings. The microscopic examination showed similar changes in all the diseased sheep but their size depended on the intensity of the Psoroptes ovis infection.

Biochemical findings. Serum MDA, TAC, TOS, and OSI levels are given in Table 1. The serum MDA, TOS, and OSI levels were significantly $(\mathrm{P}<0.01)$ increased in the psoroptic mange group compared to the healthy group. However, the serum TAC level was significantly $(\mathrm{P}<0.01)$ decreased in infected animals compared to uninfected specimens.

Correlation analysis results between TAC, TOS, OSI, and MDA in the psoroptic mange group are given in Table 2. A significantly $(\mathrm{P}<0.01)$ negative correlation between TAC and OSI, and a significantly $(\mathrm{P}<0.01)$ positive correlation between TOS and OSI were determined.

\section{Discussion}

Oxidative stress caused by excess free radical production has been determined to play a role in the pathogenesis of a number of diseases in buffalo, camels, sheep, and goats caused by sarcoptic mange $(11,12,19,34)$, and in sheep infected with psoroptic mange (14). No study relating the measurements of serum MDA, TAC, TOS, and OSI levels in sheep naturally infected with Psoroptes ovis was found. For this reason, this study was conducted to measure serum MDA, TAC, TOS, and OSI levels and evaluate the oxidative stress status in sheep naturally infected with Psoroptes ovis.

Lipids are the most susceptible substrates to free radical damage. MDA is the last product of lipid peroxidation, and is a key marker of oxidative stress (9). Various studies have reported that lipid peroxidation products, such as MDA, increase in diseases such as theileriosis in sheep (4) and coccidiosis (37) and transportation (2) in cattle. There are also other studies related to the evaluation of oxidative stress in ruminant skin diseases. A significant increase in MDA levels was reported in animals infected with sarcoptic mange: in camels (34), in sheep (19), and in buffalo liver (12). Likewise, a noteworthy rise in MDA level was observed in erythrocytes of sheep with psoroptic mange (13). In the study presented here, serum MDA levels significantly increased in sheep naturally infected with Psoroptes ovis compared to healthy sheep, which is consistent with the reports cited above. These increases were interpretable as a marker of excess free radical production in sheep with psoroptic mange.

There are numerous different antioxidant components in serum and tissues. Measurement of each antioxidant component for the determination of antioxidant status is difficult. In addition, because of coadjuvancy of different antioxidants, the determination of only one antioxidant cannot reflect their combined effects in the correct way (7). Erel (16) and $\mathrm{Cao}$ and Prior (7) have reported that TAC measurement may provide enough data to indicate 
plasma oxidant/antioxidant balance. There are numerous studies in which TAC was evaluated in ruminants. It was reported that TAC level significantly decreases in cows with bovine herpes virus-1 (14), foot and mouth disease (38), reticuloperitonitis traumatica (3), and coccidiosis (37). The serum TAC levels were found to be lower in sheep naturally infected with pox virus in the study of Kirmizigul et al. (22) and in sheep and lambs with cystic echinococcosis in the study of Ozturk et al. (28). No study related to TAC levels in ruminants with skin diseases was known to have been carried out. In the present study, serum TAC level was found to significantly decrease in sheep infected with Psoroptes ovis compared to healthy sheep. As is consistent with the above mentioned reports, this condition was interpreted to mean that antioxidants might have been used to scavenge free radicals produced during infection. This interpretation was supported by the negative correlation between TAC and OSI in sheep infected with Psoroptes ovis.

Because one-by-one measurement of oxidant molecules is impractical, use may be made of TOS level as another parameter to evaluate oxidative stress (16). Various studies have evaluated oxidative stress status using TOS level and have found discrepant results. Namely, Cingi et al. (10) reported that rectal palpation in cows caused oxidative stress, and the development of oxidative stress was attributed to increased TOS level. Durgut et al. (14) determined that there was no statistical significance to TOS level in cows with bovine herpes virus-1 but there was a negative correlation between TAC and TOS. Bozukluhan et al. (6) found that TOS level significantly increased in cows with foot and mouth disease. Kirmizigul et al. (22) found that TOS levels were increased in sheep naturally infected with pox virus. No study relating to TOS levels in skin diseases of ruminants was found. In this study the serum TOS level was determined to be significantly increased in sheep infected with Psoroptes ovis compared to healthy sheep. The increased TOS level was attributed to excessive production of free radicals during the disease course, indicating oxidative stress development. The increased MDA, significant positive correlation between TOS and OSI, and significant negative correlation between TAC and OSI in sheep infected with Psoroptes ovis support this result.

In extremely high ROS production, antioxidant enzymes are also increased to protect the structural and functional integrity of the body (36). For this reason, testing TAC and TOS levels may be insufficient for the full determination of oxidative stress in the body. Oxidative stress index is a key factor for the determination of oxidative stress (9). In determining the oxidative stress in ruminants, various studies have been carried out to evaluate OSI and contradictory data have been obtained. Durgut et al. (14) stated that there was no significant change in OSI in cows with bovine herpes virus-1 compared to healthy cows. Hanedan et al. (20) determined that serum OSI levels increased in cattle with lung cystic echinococcosis compared to healthy cattle. Abuelo et al. (1) reported that oxidative stress occurred in cows after calving and OSI was a valuable parameter to evaluate the stress. In this study, serum OSI level significantly increased in sheep infected with Psoroptes ovis compared to healthy sheep and it was concluded that OSI is a valuable parameter by which to evaluate oxidative stress. Such a conclusion is consistent with the report of Abuelo et al. (1).

The obtained results indicate that there is a possible relationship between oxidant/antioxidant imbalance and Psoroptes ovis infestation in sheep. Markers such as MDA, TAC, TOS, and OSI can be used to determine the oxidative stress in sheep naturally infected with Psoroptes ovis. Oxidative stress should also be taken up in further studies with the aim of establishing treatment procedure and facilitating prognosis of this disease.

Conflict of Interests Statement: The authors declare that there is no conflict of interests regarding the publication of this article.

Financial Disclosure Statement: The publication was financed from the authors' own funds.

Animal Rights Statement: The authors declare that the experiments on animals were conducted in accordance with local Ethical Committee laws and regulations as regards care and use of laboratory animals.

\section{References}

1. Abuelo A., Hernandez J., Benedito J.L., Castillo C.: Oxidative stress index (OSI) as a new tool to assess redox status in dairy cattle during the transition period. Animal 2013, 7, 1374-1378.

2. Aktas M.S., Ozkanlar S., Karakoc A., Akcay F., Ozkanlar Y.: Efficacy of vitamin E+selenium and vitamin $\mathrm{A}+\mathrm{D}+\mathrm{E}$ combinations on oxidative stress induced by long-term transportation in Holstein dairy cows. Livestock Sci 2011, 141, 76-79.

3. Atakisi E., Bozukluhan K., Atakisi O., Gokce H.I.: Total oxidant and antioxidant capacities and nitric oxide levels in cattle with traumatic reticuloperitonitis. Vet Rec 2010, 167, 908-909.

4. Baghshani H., Razmi G.R., Yaghfouri S., Dezaki A.A.: Status of some oxidative stress biomarkers in sheep naturally infected with theileriosis. Res Opin Anim Vet Sci 2011, 1, 499-504.

5. Baz K., Cimen M.Y.B., Koktürk A., Yazıcı A.C., Eskandari G., Ikizoglu G., Api H., Atik U.: Oxidant/antioxidant status in patients with psoriasis. Yonsei Med J 2003, 44, 987-990.

6. Bozukluhan K., Atakisi E., Atakisi O.: Nitric oxide levels, total antioxidant and oxidant capacity in cattle with foot-and-mouthdisease. Kafkas Univ Vet Fak Derg 2013, 19, 179-181.

7. Cao G., Prior R.L.: Comparison of different analytical methods for assessing total antioxidant capacity of human serum. Clin Chem 1998, 44, 1309-1315.

8. Castillo C., Hernandez J., Bravo A., Lopez-Alonso M., Pereira V., Benedito J.L.: Oxidative status during late pregnancy and early lactation in dairy cows. Vet J 2005, 169, 286-292.

9. Celi P.: The role of oxidative stress in small ruminants' health and production. R Bras Zootec 2010, 39, 348-363. 
10. Cingi C.C., Baser D.F., Karafakioglu Y.S., Fidan A.F.: Stress response in dairy cows related to rectal examination. Acta Sci Vet 2012, 40, 1053.

11. De U.K., Dey S.: Evaluation of organ function and oxidant/antioxidant status in goats with sarcoptic mange. Trop Anim Health Prod 2010, 42, 1663-1668.

12. Dimri U., Sharma M.C., Swarup D., Ranjan R., Kataria M.: Alterations in hepatic lipid peroxides and antioxidant profile in Indian water buffaloes suffering from sarcoptic mange. Res Vet Sci 2008, 85, 101-105.

13. Dimri U., Sharma M.C., Yamdagni A., Ranjan R., Zama M.M.S.: Psoroptic mange infestation increases oxidative stress and decreases antioxidant status in sheep. Vet Parasitol 2010, 68, 318-322.

14. Durgut R., Ataseven V.S., Sagkan-Ozturk A., Ozturk O.H.: Evaluation of total oxidative stress and total antioxidant status in cows with natural bovine herpesvirus-1 infection. J Anim Sci 2013, 91, 3408-3412.

15. Ercan N., Fidanci U.R.: Urine 8-hydroxy-2'-deoxyguanosine (8-OHdG) levels of dogs in pyoderma. Vet J Ankara Univ 2012, 59, 163-168.

16. Erel O.: A novel automated direct measurement method for total antioxidant capacity using a new generation, more stable ABTS radical cation. Clin Biochem 2004, 37, 277-285.

17. Erel O.: A new automated colorimetric method for measuring total oxidant status. Clin Biochem 2005, 38, 1103-1111.

18. Esmaeilnejad B., Tavassoli M., Asr1-Rezaei S., Dalır-Naghadeh B., Malekinejad H., Jalilzadeh-Amin G., Arjmand J., Golabi M., Hajipour N.: Evaluation of antioxidant status, oxidative stress and serum trace mineral levels associated with Babesia ovis parasitemia in sheep. Vet Parasitol 2014, 205, 38-45.

19. Gurgoze S.Y., Sahin T., Sevgili M., Ozkutlu Z., Ozan S.T.: The effects of ivermectin or doramectin treatment on some antioxidant enzymes and the level of lipid peroxidation in sheep with natural sarcoptic scap. J Vet Med Yüzüncü Yıl Univ 2003, $14,30-34$.

20. Hanedan B., Kirbas A., Kandemir F.M., Ozkaraca M., Kilic K., Benzer F.: Arginase activity and total oxidant/antioxidant capacity in cows with lung cystic echinococcosis. Med Weter $2015,71,167-170$

21. Kataria A.K., Kataria N.: Evaluation of oxidative stress in sheep affected with Peste des petits ruminants. J Stress Physiol Biochem 2012, 8, 73-77.

22. Kırmızıgul A.H., Ogun M., Ozen H., Erkilic E.E., Gokce E., Karaman M., Kukurt A.: Oxidative stress and total sialic acid levels in sheep naturally infected with pox virus. Pakistan Vet $\mathrm{J}$ 2016, 36, 312-315.

23. Kleczkowski M., Klucinski W., Sikora J., Zdanowicz M., Dziekan P.: Role of antioxidants in the protection against oxidative stress in cattle nonenzymatic mechanism. Pol J Vet Sci 2003, 6, 301-308.

24. Kocyigit A., Keles H., Selek S., Guzel S., Celik H., Erel O.: Increased DNA damage and oxidative stress in patients with cutaneous leishmaniasis. Mutat Res 2005, 585, 71-78.

25. Kosecik M., Erel O., Sevinc E., Selek S.: Increased oxidative stress in children exposed to passive smoking. Int $\mathrm{J}$ Cardiol 2005, 100, 61-64.

26. Lykkesfeldt J., Svendsen O.: Oxidants and antioxidants in disease: oxidative stress in farm animals. Vet $\mathrm{J}$ 2007, 173, 502-511.

27. Mutlu B., Aksoy N., Cakir H., Celik H., Erel O.: The effects of the mode of delivery on oxidative-antioxidative balance. J. Matern Fetal Neonatal Med 2011, 24, 1367-1370.

28. Ozturk A.S., Durgut R., Ozturk O.H.: Oxidant/antioxidant status in lambs and sheep with liver and lung cystic echinococcosis diagnosed by ultrasonography and necropsy. Vet Parasitol 2015, 208, 280-285.

29. Pastore S., Korkina L.: Redox imbalance in T cell-mediated skin diseases. Mediators Inflamm 2010, 2010, 1-9.

30. Piccione G., Casella S., Giannetto C., Bazzano M., Giudice E., Fazio F.: Oxidative stress associated with road transportation in ewes. Small Rum Res 2013, 112, 235-238.

31. Placer Z.A., Chusman L., Johnson B.C.: Estimation of products of lipid peroxidation in biological fluids. Anal Biochem 1966, $16,359-364$.

32. Radostits O.M., Gay C.C., Hinchcliff K.W., Constable P.D.: Veterinary Medicine. W.B. Saunders, London. 2006, pp. 1610-1611.

33. Roberts I.H., Meleney W.P.: Variations among strains of Psoroptes ovis (Acarina; Psoroptidae) on sheep and cattle. Ann Entomol Soc Am 1971, 64, 109-116.

34. Saleh M.A., Mahran O.M., Al-Salahy M.B.: Circulating oxidative stress status in dromedary camels infested with sarcoptic mange. Vet Res Commun 2011, 35, 35-45.

35. Stewart T.G.B., Tom N.M., Craig A.W., Alasdair J.N., John F.H.: Host transcription factors in the immediate proinflammatory response to the parasitic mite Psoroptes ovis. Plos One 2011, 9, e24402.

36. van den Broek A.H., Huntley J.F.: Sheep scab: the disease, pathogenesis and control. J Comp Pathol 2003, 128, 79-91.

37. Yilmaz S., Issi M., Kandemir F.M., Gul Y.: Malondialdehyde and total antioxidant levels and hematological parameters of beef cattle with coccidiosis. J Vet Med Yüzüncü Y1l Univ 2014, 25, 41-45.

38. Zaher K.S., Ahmed W.M.: Impact of foot and mouth disease on oxidative status and ovarian activity in Egyptian buffaloes. World J Zool 2008, 3, 1-7. 